\title{
High-Precision Values of the Gamma Function and of Some Related Coefficients
}

\author{
By Arne Fransén and Staffan Wrigge
}

\begin{abstract}
In this paper we determine numerical values to $80 \mathrm{D}$ of the coefficients in the Taylor series expansion $\Gamma^{m}(s+x)=\Sigma_{0}^{\infty} g_{k}(m, s) x^{k}$ for certain values of $m$ and $s$ and use these values to calculate $\Gamma(p / q)(p, q=1,2, \ldots, 10 ; p<q)$ and $\min _{x>0} \Gamma(x)$ to $80 \mathrm{D}$. Finally, we obtain a high-precision value of the integral $\int_{0}^{\infty}(\Gamma(x))^{-1} d x$.
\end{abstract}

1. Introduction. This paper traces its origin from a wish to determine highprecision values of the integrals $F=\int_{0}^{\infty}(\Gamma(x))^{-1} d x$ and $\int_{n}^{n+1}(\Gamma(x))^{-1} d x$ because the distribution defined by $G(x)=F^{-1} \int_{0}^{x}(\Gamma(t))^{-1} d t$ may be useful in reliability theory (Fransén [5]). That can also be approximated by a weighted sum of Gamma Distributions or be seen as a special case of using the Fox $H$-function as a distribution (Carter and Springer [3]). The integrals in question had not been properly studied (to our great astonishment) before. They were not even mentioned in Nielsen's book on the Gamma function [11]. The closest we came in our literature study was to a paper from 1883 by Bourguet [2]. When establishing high-precision values of the integrals Fransén [4] had to calculate the Riemann Zeta function to 80D for integer values, thereby using formulas of Katayama and Ramanujan [8]. These values might be used for many purposes: to determine the coefficients in the Taylor series expansion of $\Gamma^{m}(s+x)$ for certain interesting values of $m$ and $s$, the value of $\min _{x>0} \Gamma(x)$, and many other relevant coefficients.

When carrying out the necessary multiple-precision calculation on our DEC-10 computer we have used a Simula Class HIGHPREC developed by a student, Demetre Betsis, at the University of Stockholm.

2. Numerical Values to $80 \mathrm{D}$ of the Coefficients in the Taylor Series Expansion of $\Gamma^{m}(s+x)$ for Certain Values of $m$ and $s$.

a. Basic Formulas. Let $m$ and $s$ be real numbers, $s>0$. Consider the Taylor series expansion

where

$$
\Gamma^{m}(s+x)=\sum_{k=0}^{\infty} g_{k}(m, s) x^{k}
$$

$$
g_{k}(m, s)=\frac{1}{k !} \frac{d^{k} \Gamma^{m}(s)}{d s^{k}} .
$$

Received April 30, 1979; revised August 14, 1979.

AMS (MOS) subject classifications (1970). Primary 65D20; Secondary 33A15, 33A25, $65 \mathrm{~A} 05$.

Key words and phrases. Special functions, Gamma function, Riemann Zeta function. 
The coefficients $g_{k}(m, s)$ may be obtained recursively if we apply Leibniz' differentiation formula to the identity

$$
\frac{d \Gamma^{m}(s)}{d s}=m \psi(s) \Gamma^{m}(s)
$$

where $\psi(s)$ is the Psi function $(=\dot{\Gamma}(s) / \Gamma(s))$. We state the main result in the following

THEOREM. Let $m$ and $s$ be real numbers, $s>0$. The coefficients $g_{k}(m, s)$ in the Taylor series expansion

$$
\Gamma^{m}(s+x)=\sum_{k=0}^{\infty} g_{k}(m, s) x^{k}
$$

are obtained from the recursion formula

$$
(n+1) g_{n+1}(m, s)=m \sum_{k=0}^{n}(-1)^{k-1} g_{n-k}(m, s) h_{k}(s),
$$

where $h_{k}(s)(k=1,2, \ldots)$ is the Hurwitz Zeta function

$$
h_{k}(s)=\sum_{n=0}^{\infty} \frac{1}{(s+n)^{k+1}}
$$

and

$$
h_{0}(s)=-\psi(s)=\lim _{n=\infty}\left(\sum_{k=0}^{n} \frac{1}{s+k}-\ln (n)\right),
$$

with the initial value $g_{0}(m, s)=\Gamma^{m}(s)$.

A similar result can partly be found in Nielsen's book [11]. We now apply the theorem to some special cases.

b. Special Cases. Choosing $s=1$, we get $g_{0}(m, 1)=1$ and $h_{0}(1)=\gamma$, the Euler constant. Further, we then have $h_{k}(1)=\zeta(k+1)$, the Riemann Zeta function, for $k=1,2, \ldots$ The computation of values of $\zeta(k)$ for even values of $k$ is straightforward, while we use the Ramanujan formula as described in [8] to compute them in the odd case. In Table I we present these values to $80 \mathrm{D}$ for $k$ up to 51.

Putting $m=-1$ and denoting $a_{k+1}=g_{k}(-1,1)$, we compute the coefficients using the recursion formula. In Table II we present the values of $a_{k}$ obtained to $80 \mathrm{D}$ for $k$ up to 52 . Note that $a_{k}$ approaches zero very fast. We prove that $\lim _{k=\infty} a_{k}=0$ below. We have not been able to derive an approximate expression of $a_{k}$ or to explain the rather irregular occurrences of plus and minus signs. Already for moderate sizes of $s$, however, the expansion is properly alternating. Similarly when choosing $m=1$ and denoting $b_{k+1}=g_{k}(1,1)$ we get the values of $b_{k}$ presented in Table III. We prove also that $\lim _{k=\infty} g_{2 k}(1,1)=1$ and $\lim _{k=\infty} g_{2 k+1}(1,1)=-1$ below.

Finally, choosing $s=3 / 2$, we get $-h_{0}(s)=\psi(s)=2-\gamma-2 \ln 2$ and $h_{k}(s)=\left(2^{k+1}-1\right) \xi(k+1)-2^{k+1}$ (see [1]) for $k=1,2, \ldots$ We denote for $m=1, c_{k+1}=g_{k}(1,3 / 2)$ and for $m=-1, d_{k+1}=g_{k}(-1,3 / 2)$; and present the 
values of $c_{k}$ and $d_{k}$ in Tables IV and V. Note that $g_{k}(1,3 / 2) \approx(-1)^{k}(2 / 3)^{k+1}$ and $\lim _{k=\infty} g_{k}(-1,3 / 2)=0$.

Some of the results presented in Tables $\mathrm{I}-\mathrm{V}$ have partly been published previously. A paper by J. W. Wrench [13] gives the coefficients $a_{k}$ to $31 \mathrm{D}$ for $k=$ 2(1)41. Last-figure corrections appeared in Math. Comp., v. 27, 1973, pp. 681-682, MTE 505. A. H. Morris has compiled two unpublished tables, deposited in the UMT file. The first one, [9], gives $\zeta(k)$ to $70 \mathrm{D}$ for $k=2(1) 90$. The second one, [10], includes a tabulation of $a_{k}$ to 70D for $k=1(1) 73$.

c. A Draft Proof. In the text (Section 2b) it is remarked that $\lim _{k=\infty} a_{k}=0$ and that $\lim _{k=\infty} g_{2 k}(1,1)=-\lim _{k=\infty} g_{2 k+1}(1,1)=1$.

By using ordinary residue calculus one sees that

$$
\frac{1}{2 \pi i} \int z^{-k} \frac{1}{\Gamma(z)} d z=a_{k-1},
$$

where the integration is carried out around $|z|=1$. We put $z=e^{i \theta}$. Then

$$
a_{k}=\frac{1}{2 \pi} \int_{0}^{2 \pi}(\cos (k \theta)-i \sin (k \theta)) \frac{1}{\Gamma\left(e^{i \theta}\right)} d \theta
$$

Using a well-known lemma of Riemann-Lebesgue, one gets $\lim _{k=\infty} a_{k}=0$. To prove the other results one starts with the identity

$$
\Gamma(s)=\int_{0}^{\infty} x^{s-1} e^{-x} d x=R_{1}(s)+R_{2}(s)
$$

where

$$
R_{1}(s)=\int_{0}^{1} x^{s-1} e^{-x} d x=\sum_{k=0}^{\infty} \frac{(-1)^{k}}{(s+k) k !}
$$

and

$$
R_{2}(s)=\int_{1}^{\infty} x^{s-1} e^{-x} d x
$$

Differentiating Eq. (2.5) $n$ times with respect to $s$, we get

$$
\Gamma^{(n)}(s) \frac{1}{n !}=\sum_{k=0}^{\infty} \frac{(-1)^{k+n}}{(s+k)^{n+1} k !}+\frac{R_{2}^{(n)}(s)}{n !} .
$$

When $s=1$ and $n \rightarrow \infty$ "everything" in the right-hand side of Eq. (2.6) approaches zero except the term $(-1)^{n}$. Q.E.D.

3. Numerical Values to $80 \mathrm{D}$ of $\Gamma(p / q) ; p, q=1,2, \ldots, 10, p<q$.

a. By Taylor Series Expansions. We shall calculate in all 32 different values $\Gamma(p / q)$, where $p / q \in I$ and $I=I_{1} \cup I_{2} \cup I_{3} \cup I_{4} \cup I_{5}$, where

$$
\begin{aligned}
& I_{1}=\left\{\frac{1}{10}, \frac{1}{9}, \frac{1}{8}, \frac{1}{7}, \frac{1}{6}\right\}, \\
& I_{2}=\left\{\frac{1}{5}, \frac{2}{9}, \frac{1}{4}, \frac{2}{7}, \frac{3}{10}\right\}, \\
& I_{3}=\left\{\frac{1}{3}, \frac{3}{8}, \frac{2}{5}, \frac{3}{7}, \frac{4}{9}, \frac{1}{2}, \frac{5}{9}, \frac{4}{7}, \frac{3}{5}, \frac{5}{8}, \frac{2}{3}\right\},
\end{aligned}
$$




$$
\begin{aligned}
& I_{4}=\left\{\frac{7}{10}, \frac{5}{7}, \frac{3}{4}, \frac{7}{9}, \frac{4}{5}\right\} \\
& I_{5}=\left\{\frac{5}{6}, \frac{6}{7}, \frac{7}{8}, \frac{8}{9}, \frac{9}{10}, \frac{1}{1}\right\} .
\end{aligned}
$$

We use the Taylor series expansions we have, viz.

$$
f(x)=\frac{1}{\Gamma(1+x)}=\sum_{k=0}^{\infty} \cdot g_{k}(-1,1) x^{k}
$$

and

$$
g(x)=\frac{1}{\Gamma(3 / 2+x)}=\sum_{k=0}^{\infty} g_{k}\left(-1, \frac{3}{2}\right) x^{k}
$$

We have to calculate $x f(x)$ and $(x+1 / 2) g(x)$ with a precision a little greater than $0.5 \cdot 10^{-80}$, whereupon we invert. Therefore, we must have

$$
\left|g_{51}(-1,1)\right| x^{52} \lesssim 0.5 \cdot 10^{-80}, \quad\left|1 / 2 g_{51}(-1,3 / 2)\right||x|^{51} \lesssim 0.5 \cdot 10^{-80} .
$$

The corresponding values of $x$ are $0<x<0.1970$ and $|x|<0.1964$. If we also allow negative values of $x$ in $f(x)$, we get $-0.1908<x<0$.

We can hereby calculate the following values:

$$
\begin{aligned}
& \text { with } x f(x) \quad \Gamma\left(\frac{p}{q}\right) \text { where } \frac{p}{q} \in I_{1}: x=\frac{p}{q} \text { in Eq. (3.1), } \\
& \text { with }(x+1 / 2) g(x) \quad \Gamma\left(\frac{p}{q}\right) \text { where } \frac{p}{q} \in I_{3}: x+1 / 2=\frac{p}{q} \text { in Eq. (3.2), } \\
& \text { with } f(x) \quad \Gamma\left(\frac{p}{q}\right) \text { where } \frac{p}{q} \in I_{5}: 1+x=\frac{p}{q} \text { in Eq. (3.1). }
\end{aligned}
$$

The values $\Gamma(p / q)$ where $p / q \in I_{2} \cup I_{4}$ cannot be calculated in this simple way. For these missing values we use the duplication formula

$$
\Gamma(2 x) \sqrt{\pi}=2^{2 x-1} \Gamma(x) \Gamma(x+1 / 2) .
$$

The 32 values of $\Gamma(p / q)$ appear in Table VII.

b. By Elliptic Integrals. As is shown by Wrigge [14]-[16] and Glasser and Wood [7] and many others, there is a close relationship between certain values of the gamma function and the complete elliptic integral $K(t)$. The easiest way of getting a good value of $K(t)$ is to use the arithmetic-geometric mean $M(t)$ (see [1]), which will give an accurate value of $K(t)$ to at least $80 \mathrm{D}$ in less than 10 steps. We have

$$
K(t)=\frac{\pi}{2 M(t)} .
$$

It is, e.g., known that (Fransén [4])

$$
\begin{gathered}
\Gamma^{2}\left(\frac{1}{4}\right)=4 \sqrt{\pi} K\left(\frac{1}{\sqrt{2}}\right) \\
\Gamma^{2}\left(\frac{1}{8}\right)=16 K(\sqrt{2}-1) 2^{-3 / 4} \Gamma\left(\frac{1}{4}\right) .
\end{gathered}
$$


By using the duplication and reflection formulas for the Gamma function (see [1]) it is possible to get easy-to-calculate expressions for all $\Gamma(p / 8), p=1,2, \ldots, 8$. Such values are presented to $80 \mathrm{D}$ in [4]. Similar results hold for $\Gamma(p / 6)$ (see [7]). The values thus calculated agree excellently with the values calculated by the Taylor series method in a. or by other methods as presented in [6].

4. Numerical Values to $80 \mathrm{D}$ of $x_{0}$ and $\Gamma\left(x_{0}\right)=\min _{x>0} \Gamma(x)$. Instead of $\min _{x>0} \Gamma(x)$ we study $\max _{x>0}(\Gamma(x))^{-1}$ and, thereby, use the Taylor series expansion

$$
g(x)=\frac{1}{\Gamma(3 / 2+x)}=\sum_{k=0}^{\infty} g_{k}\left(-1, \frac{3}{2}\right) x^{k} .
$$

By making a more and more refined tabulation of $g(x)$ we managed to get a value of $x_{0}$ to $37 \mathrm{D}$ and of $\min \Gamma(x)$ to $74 \mathrm{D}$. In order to get a better value of $x_{0}$ we studied

$$
\dot{g}(x)=\sum_{k=1}^{\infty} k g_{k}\left(-1, \frac{3}{2}\right) x^{k-1},
$$

and then made use of "repeated" inverse Bessel interpolation. We thus got

$$
x_{0}=1.46163214496836234126
$$

$$
26595423257213284681
$$

96204006446351295988

40859878644035380181

and

$$
\begin{aligned}
& \Gamma\left(x_{0}\right)=0.88560319441088870027 \\
& 88159005825887332079 \\
& 51533669903448871200 \\
& 16587513622741739635 \text {. }
\end{aligned}
$$

5. Numerical Values to $60 \mathrm{D}$ of $\int_{n}^{n+1}(\Gamma(x))^{-1} d x(n=-10,-9, \ldots, 48)$ and $\int_{0}^{\infty}(\Gamma(x))^{-1} d x$. The original problem for this paper was to determine a high-precision value of the integral $F=\int_{0}^{\infty}(\Gamma(x))^{-1} d x$ (named Fransén's constant in H. P. Robinson's extensive file of mathematical constants). In [4] Fransén did that using the EulerMaclaurin formula and got the value of $F$ to 65D. Here we will use the Taylor series methods in Section 2 to calculate $\int_{n}^{n+1}(\Gamma(x))^{-1} d x$ for $n \geqslant 0$.

Using routine manipulation, we get

$$
\begin{aligned}
\int_{n}^{n+1}(\Gamma(x))^{-1} d x & =\int_{0}^{1 / 4}(\Gamma(x+n))^{-1} d x \\
& +\int_{-1 / 4}^{+1 / 4}(\Gamma(x+n+1 / 2))^{-1} d x \\
& +\int_{-1 / 4}^{0}(\Gamma(x+n+1))^{-1} d x
\end{aligned}
$$


But

$$
\int_{v}^{u}(\Gamma(s+x))^{-1} d x=\sum_{k=0}^{\infty} g_{k}(-1, s) \frac{u^{k+1}-v^{k+1}}{k+1}
$$

Using the methods in Section 2 and/or some simpler recurrence relations, the coefficients $g_{k}(-1, n)$ and $g_{k}(-1,3 / 2+n)$ were calculated for the necessary values of $n$ and the integration carried out. The results to 60D appear in Table VI.

To calculate $\int_{n}^{n+1}(\Gamma(x))^{-1} d x$ for $n<0$ we used Eq. (5.1) valid also for $n<0$. Furthermore,

$$
\frac{1}{\Gamma(-n+x)}=x(x-1)(x-2) \ldots(x-n) \frac{1}{\Gamma(1+x)}
$$

and

$$
\frac{1}{\Gamma(-n+1 / 2+x)}=(1 / 2+x)(-1 / 2+x) \ldots(-n+1 / 2+x) \frac{1}{\Gamma\left(\frac{3}{2}+x\right)}
$$

Using the Eqs. (5.3), (5.4) and the Taylor series expansions of $(\Gamma(1+x))^{-1}$ and $(\Gamma(3 / 2+x))^{-1}$ we calculated the integrals also for negative values of $n$. The results to $60 \mathrm{D}$ are presented in Table VI.

6. Tables. In this section we present the tables previously mentioned, i.e.,

I. The values of $\gamma$, the Euler constant and $\zeta(k), k=2,3, \ldots, 51$, the Riemann Zeta function, to 80D.

II. The coefficients $g_{k}(-1,1)$ in the Taylor series expansion $(\Gamma(1+x))^{-1}=$ $\Sigma_{0}^{\infty} g_{k}(-1,1) x^{k}$ to 80D. Note that $a_{k+1}=g_{k}(-1,1)$.

III. The coefficients $g_{k}(1,1)$ in the Taylor series expansion $\Gamma(1+x)=$ $\Sigma_{0}^{\infty} g_{k}(1,1) x^{k}$ to 80D. Note that $b_{k+1}=g_{k}(1,1)$.

IV. The coefficients $g_{k}(1,3 / 2)$ in the Taylor series expansion $\Gamma(3 / 2+x)=$ $\Sigma_{0}^{\infty} g_{k}(1,3 / 2) x^{k}$ to 80D. Note that $c_{k+1}=g_{k}(1,3 / 2)$.

V. The coefficients $g_{k}(-1,3 / 2)$ in the Taylor series expansion $(\Gamma(3 / 2+x))^{-1}=$ $\Sigma_{0}^{\infty} g_{k}(-1,3 / 2) x^{k}$ to 80D. Note that $d_{k+1}=g_{k}(-1,3 / 2)$.

VI. The values of $\int_{0}^{\infty}(\Gamma(x))^{-1} d x$ and $\int_{n}^{n+1}(\Gamma(x))^{-1} d x(n=-10,-9, \ldots, 48)$ to $60 \mathrm{D}$.

VII. The values of $\Gamma(p / q), p, q=1,2, \ldots, 10: p<q$, to 80D.

Tabulated values are commonly rounded with a last-figure error not exceeding half a unit.

Acknowledgements. For valuable support during this work we want to thank Dr. John W. Wrench, Jr. and Herman P. Robinson. 


\section{TABLE I}

Values of the Euler constant, Gamma, and the Riemann Zeta function for integral values.

Carma $=0.57721566490153286060651209008240243104215933593992359880576723488486772677766467$

Zeta( 2) $=1.64493406684822643647241516664602518921894990120679843773555822937000747040320087$ Zeta( 3) $=1.20205690315959428539973816151144999076498629234049888179227155534183820578631309$ Zeta( 4$)=1.08232323371113819151600369654116790277475095191872690768297621544412061618696885$ Zeta( 5$)=1.03692775514336992633136548645703416805708091950191281197419267790380358978628148$ $\operatorname{Zeta}(6)=1.01734306198444913971451792979092052790181749003285356184240866400433218290195790$ Zeta( 7) $=1.00834927738192282683979754984979675959986356056523870641728313657160147831735574$ Zetal 8$)=1.00407735619794433937868523850865246525896079064985002032911020265258295257474881$ Zeta( 9$)=1.00200839282608221441785276923241206048560585139488875654859661590978505339025840$ Zeta(10) $=1.00099457512781808533714595890031901700601953156447751725778899463629146515191295$ Zeta(11) $=1.00049418860411946455870228252646993646860643575820861711914143610005405979821981$ Zeta(12) $=1.000246086553308048298637998047739670960416088,45800340453304095213325201968194091$ Zeta(13) $=1.00012271334757848914675183652635739571427510589550984513670267162089672682984421$ Zeta(14) $=1.00006124813505870482925854510513533374748169616915454948275520225286294102317742$ Zeta(15) $=1.00003058823630702049355172851064506258762794870685817750656993289333226715634228$ Zeta(16) $=1.00001528225940865187173257148763672202323738899047153115310520358878708702795315$ Zeta(17) $=1.0000076371976378997622736002935630292130882490925267909537984397293564329028246$ Zeta(18) $=1.00000381729326499983985646164462193973045469721895333114317442998763003954265005$ Zeta(19) $=1.00000190821271655393892565695779510135325857114483863023593304676182394970534131$ Zeta(20) $=1.00000095396203387279611315203868344934594379418741059575005648985113751373114390$ Zeta(21) $=1.00000047693298678780646311671960437304596644669478493760020748737659683908789816$ Zeta(22) $=1.00000023845050272773299000364818675299493504182177965826984960311647445893562291$ Zeta(23) $=1.00000011921992596531107306778871888232638725499778451985860322579723624373042744$ Zeta(24) $=1.00000005960818905125947961244020793580122750391883730279586424697232172449535547$ $\operatorname{Zeta}(25)=1.00000002980350351465228018606370506936601184473091954331239868133901338446076746$ Zeta(26) $=1.00000001490155482836504123465850663069862886478816788591054743596878997129674486$ Zeta(27) $=1.00000000745071178983542949198100417060411945471903188256582999323957835214760627$ Zete(28) $=1.00000000372533402478845705481920401840242323289305929581151976933470616960496030$ Zeta(29) $=1.00000000186265972351304900640390994541694806166533046920066577489380555809169327$ Zeta(30) $=1.00000000093132743241966818287176473502121981356795513681618500861336044196067294$ Zeta(31) $=1.00000000046566290650337840729892332512200710626918533694730737297169337117566989$ Zeta(32) $=1.00000000023283118336765054920014559759404950248298228453031107760225838791218939$ Zeta(33) $=1.00000000011641550172700519775929738354563095165224717276359325651773994702912462$ Zeta(34) $=1.000000000058207720879027008892436859891063054173122604617215955071688124163$ of 140 $\operatorname{Zeta}(35)=1.00000000002910385044497099686929425227884046410698196743303225621025482564048890$ Zeta(36) $=1.00000000001455192189104198423592963224531842098380889412403806913954221857174587$ $\operatorname{Zeta}(37)=1.00000000000727595983505748101452086901233805926485092555546610770579694263843837$ Zeta(38) $=1.00000000000363797954737865119023723635587327351264602838489746994795159404271425$ Zeta(39) $=1.00000000000181898965030706594758483210073008503058930961866407053525125335650932$ Zeta(40) $=1.00000000000090949478402638892825331183869490875386000099087882850547971011202537$ Zeta(41) $=1.00000000000045474737830421540267991120294885703390452991143862808123403590500260$ Zeta(42) $=1.00000000000022737368458246525152268215779786912138298219891587258053364788222296$ Zeta(43) $=1.00000000000011368684076802278493491048380259064374359028425179989901227630935911$ $\operatorname{Zeta}(44)=1.00000000000005684341987627585609277182967524068553057158899388351630644444046359$ Zeta(45) $=1.00000000000002842170976889301855455073704942662074368826530983382762906278175669$ $\operatorname{Zeta}(46)=1.00000000000001421085482803160676983430714173953767869860563395195745170024420901$ Zeta(47) $=1.00000000000000710542739521085271287735447995680002274204359368768836382888775095$ $\operatorname{Zeta}(48)=1.00000000000000355271369133711367329846953405934299214565550306261501251793404758$ Zeta(49) $=1.00000000000000177635684357912032747334901440027957015550857532695197875924123327$ Zeta(50) $=1.0000000000000008881784210930815903096091386391386325608 \quad 8714646446664497698990084$ Zeta(51) $=1.00000000000000044408921031438133641977709402681213364596030702441802859783115341$ 
TABLE II

Values of the coefficients in the expansion of the inverted Gamma function for $s=1$.

$a(1)=1.00000000000000000000000000000000000000000000000000000000000000000000000000000000$ $a(2)=0.57721566490153286060651209008240243104215933593992359880576723488486772677766467$ $a(3)=-0.65587807152025388107701951514539048127976638047858434729236244568387083835372210$ $a(4)=-0.04200263503409523552900393487542981871139450040110609352206581297618009687597599$ $a(5)=0.16653861138229148950170079510210523571778150224717434057046890317899386605647425$ $a(6)=-0.04219773455554433674820830128918739130165268418982248637691887327 \quad 545901118558900$ $a(7)=-0.00962197152787697356211492167234819897536294225211300210513886262731167351446074$ $a(8)=0.00721894324666309954239501034044657270990480088023831800109478117362259497415854$ $a(9)=-0.00116516759185906511211397108401838866680933379538405744340750527562002584816653$ $a(10)=-0.00021524167411495097281572996305364780647824192337833875035026748908563946371678$ $a(11)=0.00012805028238811618615319862632816432339489209969367721490054583804120355204347$ $a(12)=-0.00002013485478078823865568939142102181838229483329797911526116267090822918618897$ $a(13)=-0.00000125049348214267065734535947383309224232265562115395981534992315749121245561$ $a(14)=0.00000113302723198169588237412962033 \quad 074494332400483862107565429550539546040842730$ $a(15)=-0.00000020563384169776071034501541300205728365125790262933794534683 \quad 172533245680371$ $a(16)=0.0000000061 \quad 160951044814158178624986828553428672758657197123208673240292 \quad 7723507435$ $a(17)=0.00000000500200764446922293005566504805999130304461274249448171895337887737472132$ $a(18)=-0.00000000118127457048702014458812656543650557773875950493258759096189263169643391$ $a(19)=0.00000000010434267116911005104915403323122501914007098231258121210871073927347588$ $a(20)=0.00000000000778226343990507125404993731136077722606808618139293881943550732692987$ $a(21)=-0.00000000000369680561864220570818781587808576623657096345136099513648454655443000$ $a(22)=0.00000000000051003702874544759790154813228632318027268860697076321173501048565735$ $a(23)=-0.00000000000002058326053566506783222 \quad 429544855237419746091080810147188058196444349$ $a(24)=-0.00000000000000534812253942301798237001731872793994898971547812068211168095493211$ $a(25)=0.00000000000000122677862823826079015889384662242242816545575045632136601135999606$ $a(26)=-0.00000000000000011812593016974587695137645868422978312115572918048478798375081233$ $a(27)=0.00000000000000000118669225475160033257977724292867407108849407966482711074006109$ $a(28)=0.00000000000000000141238065531803178155580394756670903708635075033452562564122263$ $a(29)=-0.00000000000000000022987456844353702065924785806336992602845059314190367014889830$ $a(30)=0.00000000000000000001714406321927337433383963370267257066812656062517433174649858$ $a(31)=0.00000000000000000000013373517304936931148647813951222680228750594717618947898583$ $a(32)=-0.0000000000000000000002054233551766672789325025351355733796 u ́ 220379352387364127301$ $a(33)=0.00000000000000000000002736030048607999844831509904330982014865311695836363370165$ $a(34)=-0.00000000000000000000000173235644591051663905742845156477979906974910879499841377$ $a(35)=-0.00000000000000000000000002360619024499287287343450735427531007926413552145370486$ $a(36)=0.000000000000000000000000018649829417172944307 \quad 18413161878666898945868429073668232$ $a(37)=-0.00000000000000000000000000221809562420719720439971691362686037973177950067567580$ $a(38)=0.00000000000000000000000000012977819749479936688244144863305941656194998646391332$ $a(39)=0.00000000000000000000000000000118069747496652840622274541550997151855968463784158$ $a(40)=-0.00000000000000000000000000000112458434927708809029365467426143951211941179558301$ $a(41)=0.00000000000000000000000000000012770851751408662039902066777511246477487720656005$ $a(42)=-0.00000000000000000000000000000000739145116961514082346128933010855282371056899245$ $a(43)=0.00000000000000000000000000000000001134750257554215760954165259469306393008612196$ $a(44)=0.00000000000000000000000000000000004639134641058722029944804907952228463057968680$ $a(45)=-0.00000000000000000000000000000000000534733681843919887507741819670989332090488591$ $a(46)=0.000000000000000000000000000000000000320799592 \quad 36133526228612372790827943910901464$ $a(47)=-0.00000000000000000000000000000000000000444582973655075688210159035212464363740144$ $a(48)=-0.00000000000000000000000000000000000000131117451888198871290105849438992219023663$ $a(49)=0.00000000000000000000000000000000000000016470333525438138868182593279063941453996$ $a(50)=-0.00000000000000000000000000000000000000001056233178503581218600561071538285049997$ $a(51)=0.00000000000000000000000000000000000000000026784429826430494783549630718908519485$ $a(52)=0.00000000000000000000000000000000000000000002424715494851782689673032938370921241$ 
TABLE III

Values of the coefficients in the expansion of the Gamma function for $s=1$.

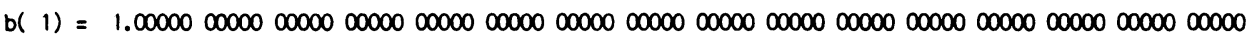
$b(2)=-0.57721566490153286060651209008240243104215933593992359880576723488486772677766467$ $b(3)=0.98905599532797255539539565150063470793918352072821409044319578368613663204947877$ $b(4)=-0.90747907608088628901656016735627511492861144907256376094133115405046518237223069$ $b(5)=0.98172808683440018733638029402185085036057367972346541540495745559385683924869345$ $b(6)=-0.98199506890314520210470141379137467551742650714719893049996719048800636496405004$ $b(7)=0.99314911462127619315386725332865849803749075523943162647824606777463099333789471$ $b(8)=-0.99600176044243153397007841966456668673529880955457891534629261939789769273081415$ $b(9)=0.99810569378312892197857540308836723752396852479018340931782415586785093718157519$ $b(10)=-0.99902526762195486779467805964888808853230396352566092666046049520894405396852065$ $b(11)=0.99951565607277744106705087759437019443450329799459966188779553794164798501358241$ $b(12)=-0.99975659750860128702584244914060923599695138562883011600298761907944923982967513$ $b(13)=0.99987827131513327572617164259000321938762910895432351359649986723614449637224390$ $b(14)=-0.99993906420644431683585223136895513185794350282804054385144515253461440778323981$ $b(15)=0.99996951776348210449861140509195350726552804247987554886576348316844865903621443$ $b(16)=-0.99998475269937704874370963172444753832608332577144871877332714971240855935928436$ $b(17)=0.999992374479073215855395094505107825833816344694662343759939159753956468407 \quad 77736$ $b(18)=-0.99999618658947331202896495779561431380201731243262962649100335457524594309637567$ $b(19)=0.99999809308113089205186619151459489773169557198830072474211716990058860564600075$ $b(20)=-0.99999904646891115771748687947054372632469616324955253563948537951581266555998870$ $b(21)=0.99999952321060573957523929299106456816809968908595024111556689661938593434377032$ $b(22)=-0.9999997615973443805709247010625874474860974860610679761 \quad 1580489247541054385393630$ $b(23)=0.99999988079601916841665041840424924052653546612259914129036430956362256423455053$ $b(24)=-0.99999994039712498374586288797675081784807034234988011151114799845552864359609524$ $b(25)=0.99999997019826754235557449619251141981339333105311537026060044530053300361141237$ $b(26)=-0.99999998509903547504708716847676946506239325845458434684290534506541463721086400$ $b(27)=0.9999999925494849624647047992537236670398874480855398289220092062180220824849029 i$ $i(28)=-0.99999999627473155543691433333928727572305682755466890354164703001136979351495990$ $b(29)=0.99999999813736213559466706281273498736621666436388532691126606099689306655138883$ $b(30)=-0.99999999906867985370787921791028525589936386514797105385555663652876112871183105$ $b(31)=0.99999999953433952214542133897633392293554270848053104176917395130246668232865762$ $b(32)=-0.999999999767169626166860999055776995711380146585384664227035399581215157182 \quad 27574$ $b(33)=0.99999999988358476811406141214433725113800419365578380133026938598690101060332961$ $b(34)=-0.99999999994179236906705284982271872517684048660470813904819976676583866719470938$ $b(35)=0.99999999997089617953682009660890584083124988584544422562183034948518816259116490$ $b(36)=-0.99999999998544808810282951070386516331145684276628909647855767215679399200977029$ $b(37)=0.99999999999272404349621830230531125595627072322128961183329384663186715187665851$ $b(38)=-0.99999999999636202156304293164652931244750676242713561002238652560976176531596127$ $b(39)=0.99999999999818101071983254219288049411381832277943535919363762116858660984978422$ $b(40)=-0.99999999999909050533935325060307999679181563265304676986551479163383166045038611$ $b(41)=0.999999999999545252662822273649383045680497889344445698331400939994148341626443744$ $b(42)=-0.99999999999977262632912635006866751576904762395962250726280105408824483514307778$ $b(43)=0.99999999999988631316380157873099034078025825870406382400268530789667979184733938$ $b(44)=-0.99999999999994315658164692375154501225136724274506101895612636214294423331851727$ $b(45)=0.99999999999997157829073883995957983221601519201567575450511667538830087214277634$ $b(46)=-0.99999999999998578914534121266317329621798543685324882725874276261924478504086160$ $b(47)=0.99999999999999289457266120388990966134103580953299726376887293563022689801663603$ $b(48)=-0.99999999999999644728632746779702797044838416549645596798092927883778119023563128$ $b(49)=0.99999999999999822364316268918236306507226616997315415572718351523402651681002934$ $b(50)=-0.999999999999999111821580996352 .42073401541651226935391984917890362574989107992032$ $b(51)=0.99999999999999955591079038209661247785982955161102781376547402255930514529416835$ $b(52)=-0.99999999999999977795539515235510420346568761595224821682619772031724660499422854$ 


\section{TABLE IV}

Values of the coefficients in the expansion of the Gamma function for $s=3 / 2$.

$c(1)=0.88622692545275801364908374167057259139877472806119356410690389492645564229551609$ $c(2)=0.03233839744888501382886988426897030778133478887050702063664101945985959916217310$ $c(3)=0.41481345368830116823003762311135634284890996337042236797771975186726615369242118$ $c(4)=-0.10729480456477221168754195638970966205457592382129830093863921109250105147065111$ $c(5)=0.14464535904462154303833221025388452407002686153098142841396813793811592186683805$ $c(6)=-0.07752305229985420344467732141650897047421612582748326899895306131910868700327711$ $c(7)=0.05861030381717628950418873781914405710555489249810416046394988584176964321284699$ $c(8)=-0.03800193555486513025205107101503415523796669262041889959833143979827090370222051$ $c(9)=0.02583760645575620389370000873664624629696256825229612494766554531456105142142953$ $c(10)=-0.01722244311346462506583068426038043069746008397127204062162285099106709344978632$ $c(11)=0.01152251539239922834772873294217459053339572140395147161792032958915992906518147$ $c(12)=-0.00769021136424157866258878661769250215938074247981015657578088757512194827984295$ $c(13)=0.00513164350191238754090720335432845989703065146507493943646231904812356620927091$ $c(14)=-0.00342280249735970609697968500486505430798080707929915217260630447001818329926725$ $c(15)=0.00228258976379026741393108053031818453527681390405826953702675690834987786982913$ $c(16)=-0.00152201007112442832081291296877465833025931602021860163191074780834757759267488$ $c(17)=0.00101478774215147788224108394850899469227404758873390393778910126353481173099948$ $c(18)=-0.00067657084106001236729184217880653689569577242457646486647373239889561511142475$ $c(19)=0.00045106552539565954882790570494617896557411298111940857805918701986615326832044$ $c(20)=-0.00030$ or 1767120056376190660288649784353763114596634487832892064831649745893443688 $c(21)=0.00020048137704905741940098201147684334533615787241633778862121677066934861250599$ $c(22)=-0.00013365542345929399547565033127853012420175585915244192569077270299042675658796$ $c(23)=0.00008910408456105664040042141226639387823933108834305643263548171944003297893219$ $c(24)=-0.00005940291063231535130122516721857783461045064335770962531008342494113406618863$ $c(25)=0.00003960201546487878363617207833491095520615062389953203758933484145822468189622$ $c(26)=-0.00002640137366248702575518808754784965321320692752777188265734241250679175313501$ $c(27)=0.00001760092778322951645297268133788278117773652187067751044867827677777644237939$ $c(28)=-0.00001173395665893706366843794059902360695065516376273884951825769327930008762999$ $c(29)=0.00000782263969404943194353387671525538404791630500559340393223113184280704440105$ $c(30)=-0.00000521509389794887371433355193104394183668635542067094482584931431136012396866$ $c(31)=0.00000347672957273591157132217161285643840500041716851493886957508518643083635189$ $c(32)=-0.00000231781983813297604703969392435150196840022859523314591928968783689284771196$ $c(33)=0.00000154521327461256091819054125142927388039218072173602850382135413302452989758$ $c(34)=-0.00000103014220275135600589201461242312745261315377416483149434995458013419686703$ $c(35)=0.00000068676147637145434506450745562177458588755205770538049404224730025264118131$ $c(36)=-0.00000045784098739586327633817182045540542649396367127967187462121695974888836072$ $c(37)=0.00000030522732618986831036865564826089033386547656618171173772017022828147017594$ $c(38)=-0.00000020348488463029655479789618473990052424874575587485109004102559166654794125$ $c(39)=0.00000013565658995501827019195237968762527194270730710929073928727861093724482109$ $c(40)=-0.00000009043772671727378630849715425605653809848512377621788903701010319328274830$ $c(41)=0.00000006029181784375384650176993898559450601335063949668586149314146628558625375$ $c(42)=-0.00000004019454524206443034552437362977370077407221334015854214733490921382753339$ $c(43)=0.00000002679636349986770104216090373569853666390711698594071398770037613986011234$ $c(44)=-0.00000001786424233530836665971133835572331231002056006851494573265373161090125641$ $c(45)=0.00000001190949489103087091346269578417729187971520638679011824265027301792886417$ $c(46)=-0.00000000793966326101736455682206567440897066948459046856305059462572103534223790$ $c(47)=0.00000000529310884081028995751108455253072822019044749856693035495064816448588413$ $c(48)=-0.00000000352873922725967874157097129668740448452906402637206577619566914390793965$ $c(49)=0.00000000235249281819424666959037576204620394144952093929177648070493506417091649$ $c(50)=-0.00000000156832854547128211664441689814943975828853737317362029860691474447785036$ $c(51)=0.00000000104555236365090181257764217211011987712791348638392262313521875746801825$ $c(52)=-0.00000000069703490910195336899126839533401420306814691812199399273231259954406742$ 


\section{TABLE V}

Values of the coefficients in the expansion of the inverted Gamma function for $s=3 / 2$.

$d(1)=1.12837916709551257389615890312154517168810125865799771368817144342128493688298683$ $d(2)=-0.04117452644528310145 \propto 24720511570419017500611389637712862511274615607955970431681$ $d(3)=-0.52665443552554447926320797284109288560326483755682298892755059489958378051492687$ $d(4)=0.17510202604393456149512261852557115015178239943893904700262573625825136103160628$ $d(5)=0.05096686024770607677469834940975961847045954320866620275843519671909629617466347$ $d(6)=-0.042155169368535600993185435843466578578106883 .49080022761238449631630438519372522$ $d(7)=0.00661289782682412727656625603054543628350181764006030743596345731657907787053574$ $d(8)=0.00212073144257293833601185279099258131267066481192915854940976582996803532544544$ $d(9)=-0.00111073025459489071711949847886160648593534562990251230843906982691383934747839$ $d(10)=0.00015235762076747687216556541867277372301005044436307145553429774169690013022408$ $d(11)=0.00002535520492381416527825281610103662269445849751440084015053067668905569918599$ $d(12)=-0.00001389680571791375602196650395717708427758175530387543348020611521460915336819$ $d(13)=0.00000215620329051417245345561623949452187611390476783064116647242925599634663859$ $d(14)=0.00000005794264054052672504226233219530715960491641741587975764532204957525298682$ $d(15)=-0.00000008913551118311116054072102332514387332832204816656833639829288404206290282$ $d(16)=0.00000001710346941591537374932041168644903318209015748466486787406656441624523533$ $d(17)=-0.000000000931368644524190156847571210399204377 \quad 10204263766353814457 \quad 121265113138061$ $d(18)=-0.00000000026804741033496625565042604128115772347782929123376949097977904658961604$ $d(19)=0.00000000007458932233316326050692751484717263251046479697436804065524736461046225$ $d(20)=-0.0000000000080128070614147 \quad 1837091842486912360879618123021976862240236041978389465$ $d(21)=-0.00000000000008382343033451854930489938063390212506663153066500298027976031532813$ $d(22)=0.00000000000016946340904320522267740949743801413022655456353559019782030790697520$ $d(23)=-0.00000000000002787575670712575208297531479649329340826810780536761794785955019168$ $d(24)=0.00000000000000186703946950653054191191884058911440534850618666982616849195371864$ $d(25)=0.00000000000000013049499008587986588177999892735780408619288268833503213885635928$ $d(26)=-0.00000000000000004858874144187786529617310840298295710659045372022356283467410040$ $d(27)=0.00000000000000000582954269245946783185998424421052157802998296525218650592744168$ $d(28)=-0.00000000000000000025929094179937838704778451425919182189569557926683986578988532$ $d(29)=-0.00000000000000000003326754010285788598714288220413887295402603708803744006446931$ $d(30)=0.00000000000000000000794496163576810539249688165457638737019056982805065019609257$ $d(31)=-0.00000000000000000000077555432884373572939025377073167723783435449419061753896607$ $d(32)=0.00000000000000000000002553373629132969579180630480153625853188942074109757030235$ $d(33)=0.00000000000000000000000427452016014717337946002920723634231060718164964864513088$ $d(34)=-0.00000000000000000000000082633813746684495056879321725142847854547491870639008468$ $d(35)=0.00000000000000000000000007108187657253397973655066504823965103124666984269682293$ $d(36)=-0.00000000000000000000000000207494638877042961245013106900124814732985613283261884$ $d(37)=-0.00000000000000000000000000032859544069948604830125913861824274687865997726697282$ $d(38)=0.00000000000000000000000000005819433908198747873747694790222503415363400831423132$ $d(39)=-0.00000000000000000000000000000470293213044989336039902262645386248962388600506248$ $d(40)=0.000000000000000000000000000000144785565158528647157100747334167371494690517 \quad 77690$ $d(41)=0.00000000000000000000000000000001597831505478678867340594054353327738351874361494$ $d(42)=-0.00000000000000000000000000000000287824717476134448591060144370749764981279976317$ $d(43)=0.00000000000000000000000000000000023008864006120204751172378927644259230804886900$ $d(44)=-0.00000000000000000000000000000000000815246985072319699590084541422715168107183108$ $d(45)=-0.00000000000000000000000000000000000048420145213897815521955333904527971027167115$ $d(46)=0.00000000000000000000000000000000000010188259041957854896466906836982748133098481$ $d(47)=-0.00000000000000000000000000000000000000841295304954034895278855482997385937363814$ $d(48)=0.0000000000000000200000000000000000000034887005836574468609851780643782804547036$ $d(49)=0.00000000000000000000000000000000000000000756310899948695778454309915411848105670$ $d(50)=-0.00000000000000000000000000000000000000000258953190695373319518160842184582888632$ $d(51)=0.00000000000000000000000000000000000000000023070239978497803696572536033527182461$ $d(52)=-0.00000000000000000000000000000000000000000001109196438522643546500356545708362269$ 
TABLE VI

Values of the integral from a to $b$ of the inverted Gamma function.

a b Value

$\begin{array}{llllllllll}-10 & -9 & 2 & 60547.7079320506 & 2430322753586920143767668 & 4821945086411424707728908\end{array}$

$\begin{array}{lllllllllllllll}-9 & -8 & -27136.66927 & 95424 & 98804 & 74304 & 17037 & 11893 & 49938 & 94902 & 74756 & 63019 & 48137 & 25963\end{array}$

$\begin{array}{llllllllllllllll}-8 & -7 & 3156.81596 & 18461 & 08044 & 20868 & 33161 & 53950 & 15210 & 52315 & 75487 & 85943 & 49217 & 51207\end{array}$

$\begin{array}{lllllllllllllll}-7 & -6 & -415.89693 & 20832 & 07590 & 12059 & 97940 & 79191 & 40021 & 67070 & 76448 & 45235 & 77151 & 79573\end{array}$

$\begin{array}{llllllllllllll}-6 & -5 & 63.1718675843 & 52479 & 94427 & 47404 & 14317 & 31537 & 90134 & 88805 & 14586 & 10927 & 46851\end{array}$

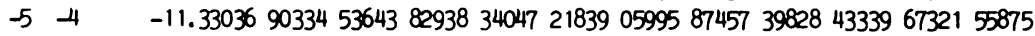

$\begin{array}{ll}-4 & -3\end{array}$

$-3-2$

$-2-1$

$\begin{array}{cc}-1 & 0\end{array}$

2.481802945521126147194193136672911756874369964282803703432296

$-0.698659909913205542697854082284220449964410869978570579228033$

0.275773033041742069460578509521115792066680306057851326554887

$-0.183720711905075623036406407517258722073681031963385069260152$

0.541235734328670530149537328879552013899256206649976097358961

1.085142664357470084326866642788474680791388749518244015155964 0.751849708495638580302403416281133892004596553910950094205304 0.311834566998537561143544732465296760762789771025220302196225 0.092018762702450046026603293339364667423662051689381530098004 $0 . œ 1046908710163388602679204887329586853659161409343951965930$ $0.003927545399260250697677505698 \quad 142145508413884542179764831450$ $0.00061871968528288900559164569032071 \quad 1002078927 \quad 145226989831581$ 0.000084310102376964410983324979051416330631927102607451396317 0.000010120467243018886953455520690961648514307663016290710441 0.000001085458619451834367184949432019038473051346836042204483 0.000000105205646890341516591603673718710348315109613127845146 $0.00000000930039695135 \quad 62725 \quad 1860374292 \quad 2218505312383772731461803$ 0.000000000755703236321622578532982536589055909970598905288909 0.000000000056809617233454611600985770508137985383930920700059 $0.00000000000397320013407821359323653 \quad 1866436230776797822257915$ 0.000000000000259782230681640553499078037948734806675093399216 0.000000000000015946579254062504276228599187165319619375066759 0.000000000000000922436110515693742638558684367054322300950137 0.000000000000000050449453403530727989469876182184099063730357 $0.00000000000000000261647493275181510998755232828011 \quad 1253685480$ 0.000000000000000000129025588783506326807476806747699108178224 0.000000000000000000006064328149315656582494574499890628073563 0.000000000000000000000272263781566489564607305974091380258658 $0.000000000000000000000011699491963783909536558 \quad 865312860394208$ 0.000000000000000000000000482070970573851132772924310311096429 0.000000000000000000000000019078960941703694107698233278372517 0.000000000000000000000000000726397872148798659987425545334646 0.000000000000000000000000000026643825660350770934578927490625 $0.00000000000000000000000000000094276 \quad 17992 \quad 14809550783363612144$ 0.000000000000000000000000000000032220458977308870213098647037 0.00000000000000000000000000000000106485534318838011727247089 0.000000000000000000000000000000000034068324048392733838593189 0.00000000000000000000000000000000000105622076381551371532268 0.000000000000000000000000000000000000031762744072759981596835 0.000000000000000000000000000000000000000927324626556745445996 0.000000000000000000000000000000000000000026306598508282595610 0.000000000000000000000000000000000000000000725712896960728736 0.000000000000000000000000000000000000000000019483252860232404 0.000000000000000000000000000000000000000000000509408787057866 0.000000000000000000000000000000000000000000000012980025713773 0.000000000000000000000000000000000000000000000000322529772329 0.000000000000000000000000000000000000000000000000007820174373 0.000000000000000000000000000000000000000000000000000185127060 0.000000000000000000000000000000000000000000000000000004281272 0.000000000000000000000000000000000000000000000000000000096774 0.000000000000000000000000000000000000000000000000000000002139 0.000000000000000000000000000000000000000000000000000000000046 0.000000000000000000000000000000000000000000000000000000000001 
TABLE VII

Values of $\operatorname{Gamma}(p / q)$.

p q Value

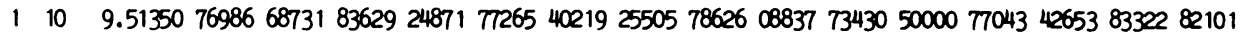

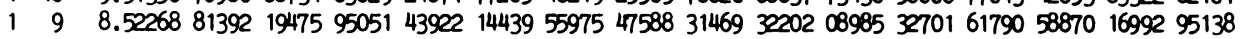

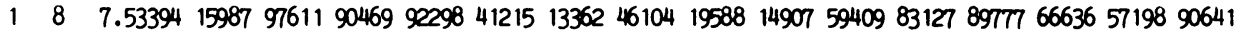

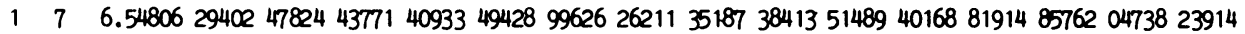

1655.56631600178023520425009689520772611139879911487285346161674462632290750281780231

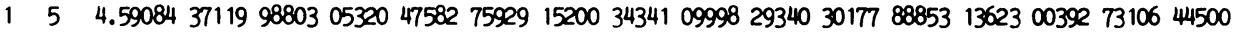

294.10657956671606119664229078891836054032105248515292424696498238258668389976146476

$1 \quad 4 \quad 3.62560990822190831193068515586767200299516768288006546743337799956991924353872912$

$27 \quad 3.14911511775993659097011366468076889222977861176625268479076150003942798453296946$

$\begin{array}{llllllll}3 & 10 & 2.991568987687590628312516515904 & 917791112806024 & 9171 & 51127 & 4411965095638876787632022\end{array}$

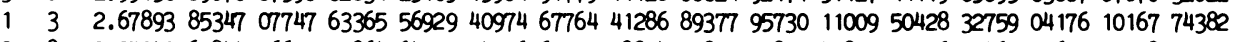

$\begin{array}{lllllllll}3 & 8 & 2.3704361844 & 1660090864 & 64735041766525098874008033589249877 & 7512693467316153135800179\end{array}$

$2 \quad 5 \quad 2.21815954375768822305905402190767945077056650177146958224197775264618516812300474$

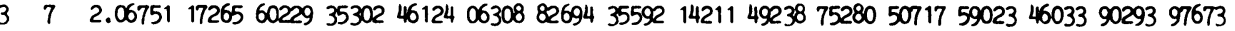

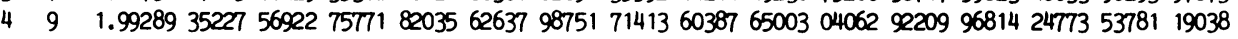

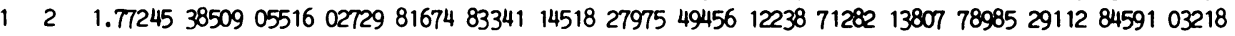

$\begin{array}{lllllllllllllll}5 & 9 & 1.60071 & 61184 & 1398328967 & 76129 & 26406 & 833697161621038 & 83996 & 53728 & 30023 & 76424 & 22664 & 60581 & 80870\end{array}$

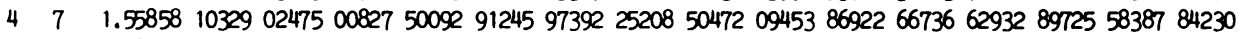

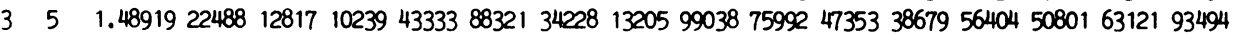

$\begin{array}{llllllll}5 & 8 & 1.434518848090556775636019739456423136632207772 & 20666733077067985809509419730209691\end{array}$

$2 \quad 3 \quad 1.35411793942640041694528802815451378551932726605679369839402246796378296540174254$

$\begin{array}{llllllllll}7 & 10 & 1.29805 & 53326 & 47557 & 78568 & 117117915281161778414117055394 & 624792164538825416815081897580\end{array}$

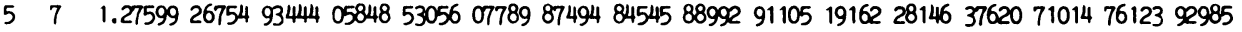

$\begin{array}{lllllll}3 & 4 & 1.225416702465177645129098303362890526851239248 & 1080706112301189382898228 & 8842679836\end{array}$

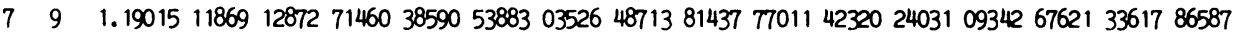

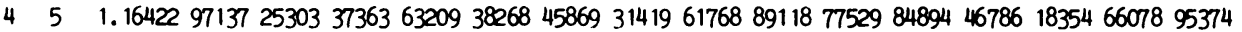

$\begin{array}{lllllll}5 & 6 & 1.12878702990812596126090109025884201332678744166475545175208351433 \quad 3770510987 & 50399\end{array}$

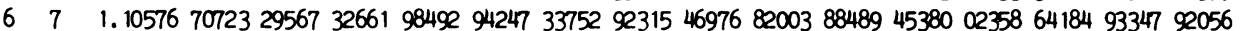

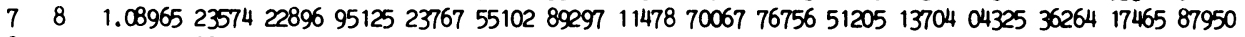

$8 \quad 9 \quad 1.07775883313349579725700633307701632920595374072476678635897539844059597442055203$

$\begin{array}{llllllllllllllll}9 & 10 & 1.0686287021 & 19319354897305335694 & 48077 & 81698 & 3878506097 & 317904937068398 & 15721 & 77025 & 41757\end{array}$

111.00000000000000000000000000000000000000000000000000000000000000000000000000000000

National Defence Research Institute

Division 1, Section 144

S-10450 Stockholm 80, Sweden

1. M. ABRAMOWITZ \& I. A. STEGUN, Handbook of Mathematical Functions with Formulas, Graphs, and Mathematical Tables, Nat. Bur. Standards, Appl. Math. Series, No. 55, U.S. Government Printing Office, Washington, D.C., 1964.

2. L. BOURGUET, "Sur les intégrales Eulériennes et quelques autres fonctions uniformes," Acta Math., v. 2, 1883, pp. 261-295.

3. B. D. CARTER \& M. D. SPRINGER, "The distribution of products, quotients and powers of independent $H$-function variates," SIAM J. Appl. Math., v. 33, 1977, pp. 542-558.

4. A. FRANSÉN, Concerning the Definite Integral $\int_{0}^{\infty}(\Gamma(x))^{-1} d x$, FOA Rapport, C10100-M4 (National Defence Research Institute, S-104 50 Stockholm 80, Sweden), 1978.

5. A. FRANSÉN, “Accurate determination of the inverse gamma integral," BIT, v. 19, 1979, pp. $137-138$.

6. D. C. GALAND \& P. F. BYRD, "High accuracy gamma function values for some rational arguments," Math. Comp., v. 22, 1968, pp. 885-887.

7. M. L. GLASSER \& V. E. WOOD, "A closed form evaluation of the elliptic integral," Math. Comp., v. 25, 1971 , pp. 535-536.

8. K. KATAYAMA, “On Ramanujan's formula for values of Riemann zeta-function at positive odd integers," Acta Arith., v. 22, 1973, pp. 149-155.

9. A. H. MORRIS, JR., "Table of the Riemann Zeta function for integer arguments," reviewed in Math. Comp., v. 27, 1973, pp. 673-674, RMT 32. 
10. A. H. MORRIS, JR., "Tables of coefficients of the Maclaurin expansions of $1 / \Gamma(z+1)$ and $1 / \Gamma(z+2)$," reviewed in Math. Comp., v. 27, 1973, p. 674, RMT 32 and RMT 33.

11. N. NIELSEN, Die Gammafunktion, Chelsea, New York, 1965.

12. H. P. ROBINSON, Private communications, 1978-1979.

13. J. W. WRENCH, JR., "Concerning two series for the gamma function," Math. Comp., v. 22, 1968, pp. 617-626.

14. S. WRIGGE, Contributions to the Theory of Elliptic Integrals, FOA P Rapport, C 8366M6 (National Defence Research Institute, S-104 50 Stockholm 80, Sweden), 1973.

15. S. WRIGGE, “An elliptic integral identity," Math. Comp., v. 27, 1973, pp. 839-840.

16. S. WRIGGE, Contributions to the Theory of Elliptic Integrals (Part 2), FOA Rapport, C10003-M6 (National Defence Research Institute, S-104 50 Stockholm 80, Sweden), 1974. 